\title{
Dislocation Density Tensor of Thin Elastic Shells at Finite Deformation
}

\section{Bohua Sun}

April 4, 2018

\begin{abstract}
The dislocation density tensors of thin elastic shells have been formulated explicitly in terms of the Riemann curvature tensor. The formulation reveals that the dislocation density of the shells is proportional to $K A^{3 / 2}$, where $K$ is the Gauss curvature and $A$ is the determinant of metric tensor of the middle surface.
\end{abstract}

Keywords dislocation density tensor, · thin shells · Riemann curvature tensor

\section{Introduction}

Fracture and elasto-plasticity of plates and shells is of great practical as well as theoretical interest[1,9-12]. For example, a large number of engineering structures, such as pressurized aircraft fuselages, ship hulls, storage tanks and pipelines are constructed of shells and plates. Concern over the safety of such structures has led to tremendous amounts of productive research in the field.

The dislocation density tensor has drawn a great deal of attention $[15$, 2,5-8] due to the fact that the dislocation is being considered as the physical mechanism of both fracture and plasticity. Recently Birsan and Neff [3] proposed a dislocation density tensor for the Cosserat theory of shells [4]. From literature survey, we noticed that the dislocation density tensors of the Mindlin-Reissner (M-R) shells [14] and Kirchhoff-Love (K-L) shells [13], have not been formulated yet. The aim of this short article is that we will apply the 3 -D dislocation density tensor formulation obtained by Sun [18] to the shell models and derived their dislocation density tensor, respectively.

Bohua Sun

Department of Mechanical Engineering, Cape Peninsula University of Technology, Bellville 7535, Cape Town, South Africa

Tel.: +27219596766

E-mail: sunb@cput.ac.za 
The paper is organized as that following an introduction, section 2 introduces the dislocation density tensor obtained by Sun [18]. Section 3 gives displacement field of thin shells. Section 4 formulates deformation gradient. Section 5 derives the dislocation density tensor for the Mindlin-Reissner and K-L theory of shells. Finally, Section 6 concludes the paper.

\section{Dislocation density tensor in terms of the Riemann curvature tensor}

In 2016 Sun [18] proved that the dislocation density tensor can be explicitly expressed by the Riemann curvature tensor, and shown that the dislocation density is proportional to the Riemann curvature tensor.

Definition 1 Let $\boldsymbol{F}$ be the deformation gradient, the definition of the dislocation tensor is given by

$$
\boldsymbol{T}=-\boldsymbol{F} \times \nabla_{\boldsymbol{X}},
$$

where, the gradient operator $\boldsymbol{\nabla}_{\boldsymbol{X}}=\frac{\partial}{\partial \boldsymbol{X}}=\frac{\partial}{\partial X^{i}} \boldsymbol{G}^{i}$ (see appendix).

Lemma 1 Let $\boldsymbol{u}$ be displacement field, $\boldsymbol{R}$ the Riemann curvature tensor, the dislocation density tensor $\boldsymbol{T}$ can be presented explicitly in terms of the Riemann curvature tensor as follows

$$
\boldsymbol{T}=-\frac{1}{2} \boldsymbol{R}(\boldsymbol{X}, \boldsymbol{Y}) \boldsymbol{u} .
$$

or in conventional form

$$
\boldsymbol{T}=-\frac{1}{2} u_{i} R_{. j k l}^{i} \boldsymbol{G}^{j} \otimes \boldsymbol{G}^{k} \times \boldsymbol{G}^{l}=-\frac{1}{2} u^{i} R_{i j k l} \boldsymbol{G}^{j} \otimes \boldsymbol{G}^{k} \times \boldsymbol{G}^{l} .
$$

The equation (2) and (3) were formulated by Sun [18].

Proof Let $\mathfrak{B}$ be undeformed configuration and $d \boldsymbol{u}=\boldsymbol{F} \cdot d \boldsymbol{Y}=\nabla_{\boldsymbol{Y}} \boldsymbol{u} \cdot d \boldsymbol{Y}$, $(\boldsymbol{X}, \boldsymbol{Y}) \in \mathfrak{B}$. According to the Stokes integration theorem, we have the displacement vector change along an arbitrary close loop as follows

$$
\begin{array}{rlc}
\boldsymbol{u} & = & \oint_{\partial \Psi} d \boldsymbol{u} \\
& = & \int_{\Psi} d(d \boldsymbol{u}) \\
& =\int_{\Psi} d\left(\boldsymbol{\nabla}_{\boldsymbol{Y}} \boldsymbol{u} \cdot d \boldsymbol{Y}\right),
\end{array}
$$

in which, $\partial \Psi$ is close boundary of a surface $\Psi \in \mathfrak{B}$, and $d \boldsymbol{u}=\nabla_{\boldsymbol{Y}} \boldsymbol{u} \cdot d \boldsymbol{Y}$ is a vector-valued 1 -form, differentiating the above equation once more, hence we obtain the vector-valued 2 -form

$$
\begin{array}{rlrl}
d\left(\nabla_{\boldsymbol{Y}} \boldsymbol{u} \cdot d \boldsymbol{Y}\right) & =d\left(\boldsymbol{\nabla}_{\boldsymbol{Y}} \boldsymbol{u}\right) \wedge d \boldsymbol{Y}+(-1)^{0} \boldsymbol{\nabla}_{\boldsymbol{Y}} \boldsymbol{u} d^{2} \boldsymbol{Y} \\
& = & \boldsymbol{\nabla}_{\boldsymbol{Y}}\left(\boldsymbol{\nabla}_{\boldsymbol{X}} \boldsymbol{u}\right) \cdot d \boldsymbol{X} \wedge d \boldsymbol{Y} \\
& = & \boldsymbol{\nabla}_{\boldsymbol{Y}} \boldsymbol{\nabla}_{\boldsymbol{X}} \boldsymbol{u} \cdot d \boldsymbol{X} \wedge d \boldsymbol{Y},
\end{array}
$$

where Poincaré Lemma [16] is used for $d^{2} \boldsymbol{Y}=0$. 
Since the antisymmetric nature of exterior algebra, $d \boldsymbol{X} \wedge d \boldsymbol{Y}=-d \boldsymbol{Y} \wedge d \boldsymbol{X}$, thus

$$
d\left(\boldsymbol{\nabla}_{\boldsymbol{Y}} \boldsymbol{u} \cdot d \boldsymbol{Y}\right)=-\frac{1}{2}\left[\boldsymbol{\nabla}_{\boldsymbol{X}} \boldsymbol{\nabla}_{\boldsymbol{Y}} \boldsymbol{u}-\boldsymbol{\nabla}_{\boldsymbol{Y}} \boldsymbol{\nabla}_{\boldsymbol{X}} \boldsymbol{u}\right] \cdot d \boldsymbol{X} \wedge d \boldsymbol{Y} .
$$

According to the definition of the Riemann operator

$$
\boldsymbol{R}(\boldsymbol{X}, \boldsymbol{Y}) \boldsymbol{u}=\boldsymbol{\nabla}_{\boldsymbol{X}} \boldsymbol{\nabla}_{\boldsymbol{Y}} \boldsymbol{u}-\boldsymbol{\nabla}_{\boldsymbol{Y}} \boldsymbol{\nabla}_{\boldsymbol{X}} \boldsymbol{u}-\boldsymbol{\nabla}_{[X, Y]} \boldsymbol{u}
$$

and in the coordinate frame, the torsion curvature $\boldsymbol{\nabla}_{[\boldsymbol{X}, \boldsymbol{Y}]} \boldsymbol{u}=0$, we have

$$
\nabla_{X} \nabla_{Y} u-\nabla_{Y} \nabla_{X} u=R(X, Y) u .
$$

If we expand the vector fields in terms of the coordinate basis $\partial_{I}$, the Riemann tensor $\boldsymbol{R}(\boldsymbol{X}, \boldsymbol{Y}) \boldsymbol{u}=\left[R_{J K L}^{I} X^{K} Y^{L} u^{J}\right] \partial_{I}$ and its components $R_{J K L}^{I}:=$ $\partial_{K} \Gamma_{L J}^{I}-\partial_{L} \Gamma_{K J}^{I}+\Gamma_{K M}^{I} \Gamma_{L J}^{M}-\Gamma_{L M}^{I} \Gamma_{K J}^{M}$. The symbols $\Gamma_{J K}^{I}$ are called the coefficients of the affine connections, or the Christoffel symbols, with respect to the frame $\boldsymbol{G}_{J}$, that is, $\boldsymbol{\nabla}_{\boldsymbol{G}_{J}} \boldsymbol{G}_{K}=\boldsymbol{G}_{I} \Gamma_{J K}^{I}$.

Therefore, we have

$$
\begin{aligned}
d\left(\boldsymbol{\nabla}_{\boldsymbol{Y}} \boldsymbol{u} \cdot d \boldsymbol{Y}\right) & =-\frac{1}{2}\left[\boldsymbol{\nabla}_{\boldsymbol{X}} \boldsymbol{\nabla}_{\boldsymbol{Y}} \boldsymbol{u}-\boldsymbol{\nabla}_{\boldsymbol{Y}} \boldsymbol{\nabla}_{\boldsymbol{X}} \boldsymbol{u}\right] \cdot d \boldsymbol{X} \wedge d \boldsymbol{Y} \\
& =\quad-\frac{1}{2} \boldsymbol{R}(\boldsymbol{X}, \boldsymbol{Y}) \boldsymbol{u} \cdot d \boldsymbol{X} \wedge d \boldsymbol{Y} .
\end{aligned}
$$

Finally, we have the displacement change in differential forms

$$
\begin{array}{ccc}
\boldsymbol{u} & = & -\int_{\Psi} d\left(\boldsymbol{\nabla}_{\boldsymbol{Y}} \boldsymbol{u} \cdot d \boldsymbol{Y}\right) \\
& =-\frac{1}{2} \int_{\Psi}\left[\boldsymbol{\nabla}_{\boldsymbol{X}} \boldsymbol{\nabla}_{\boldsymbol{Y}} \boldsymbol{u}-\boldsymbol{\nabla}_{\boldsymbol{Y}} \boldsymbol{\nabla}_{\boldsymbol{X}} \boldsymbol{u}\right] \cdot d \boldsymbol{X} \wedge d \boldsymbol{Y} \\
& =\quad-\frac{1}{2} \int_{\Psi} \boldsymbol{R}(\boldsymbol{X}, \boldsymbol{Y}) \boldsymbol{u} \cdot d \boldsymbol{X} \wedge d \boldsymbol{Y} .
\end{array}
$$

Note that the area element $d \boldsymbol{A}=d \boldsymbol{X} \wedge d \boldsymbol{Y}$, hence the dislocation density tensor $\boldsymbol{T}$ and the incompatibility operator $\operatorname{Inc}(\boldsymbol{F})$

$$
\boldsymbol{T}=\operatorname{Inc}(\boldsymbol{F})=\frac{d \boldsymbol{u}}{d \boldsymbol{A}}=-\frac{1}{2} \boldsymbol{R}(\boldsymbol{X}, \boldsymbol{Y}) \boldsymbol{u} .
$$

The proof is complete.

Equations (2) and (3) provide a general framework on formulation of dislocation density tensor for a given displacement field $\boldsymbol{u}$, especially in the case of beams, plates and shells, where the displacement field are always assumed with constraints.

\section{Displacement field of thin shells}

Let $\boldsymbol{X}=\boldsymbol{P}+\xi \boldsymbol{A}_{3}$ be position vector in the undeformed state, and $\boldsymbol{x}=\boldsymbol{p}+\xi \boldsymbol{\eta}$ be position vector in the deformed state, where the unit normal vector $\boldsymbol{A}_{3}$ is orthogonal to the undeforemd middle surface, however, in general $\boldsymbol{\eta}$ is neither unit or normal to the deformed middle surface.

The arbitrary displacement vector $\boldsymbol{u}=\boldsymbol{x}-\boldsymbol{X}=(\boldsymbol{p}+\xi \boldsymbol{\eta})-\left(\boldsymbol{P}+\xi \boldsymbol{A}_{3}\right)=$ $(\boldsymbol{p}-\boldsymbol{P})+\xi\left(\boldsymbol{\eta}-\boldsymbol{A}_{3}\right)=\boldsymbol{v}+\xi \boldsymbol{d}$, where the middle surface displacement $\boldsymbol{v}=\boldsymbol{p}-\boldsymbol{P}$, and rotation vector $\boldsymbol{d}=\boldsymbol{\eta}-\boldsymbol{A}_{3}$. 
The base vectors in the undeformed state $\boldsymbol{G}_{3}=\boldsymbol{X}_{, \xi}=\boldsymbol{A}_{3}$, and $\boldsymbol{G}_{\alpha}=$ $\boldsymbol{X}_{, \alpha}=\boldsymbol{P}_{, \alpha}+\xi \boldsymbol{A}_{3, \alpha}=\boldsymbol{A}_{\alpha}+\xi \boldsymbol{A}_{3, \alpha}=\boldsymbol{A}_{\alpha}-\xi B_{\alpha}^{\beta} \boldsymbol{A}_{\beta}=\mu_{\alpha}^{\beta} \boldsymbol{A}_{\beta}=\mu_{\alpha \beta} \boldsymbol{A}^{\beta}$, where the shitter tensor $\mu_{\alpha \beta}=A_{\alpha \beta}-\xi B_{\alpha \beta}, \mu_{\alpha}^{\beta}=\delta_{\alpha}^{\beta}-\xi B_{\alpha}^{\beta}$. The metric tensor $G_{\alpha \beta}=\mu_{\alpha}^{\lambda} \mu_{\lambda \beta}=A_{\lambda \rho} \mu_{\alpha}^{\lambda} \mu_{\beta}^{\rho}, G_{\alpha 3}=0, G_{33}=1$.

The base vectors in the deformed state $\boldsymbol{g}_{\alpha}=\boldsymbol{x}_{, \alpha}=\boldsymbol{p}_{, \alpha}+\xi \boldsymbol{\eta}_{, \alpha}=\boldsymbol{a}_{, \alpha}+\xi \boldsymbol{\eta}_{, \alpha}$ and $\boldsymbol{g}_{3}=\boldsymbol{x}_{, \xi}=\boldsymbol{\eta}$.

3.1 The displacement field of the Kirchhoff-Love theory of shells

The Kirchhoff-Love (K-L) theory of shells is an extension of Euler-Bernoulli beam theory and was developed in 1888 by Love [13] using assumptions proposed by Kirchhoff.

The K-L kinematic assumptions are: 1. straight lines normal to the midsurface remain straight after deformation; 2 . straight lines normal to the midsurface remain normal to the mid-surface after deformation; and 3. the thickness of the plate does not change during a deformation.

For the Kirchhoff-Love theory of shells [13], the displacement of thin shells can be represented as $\boldsymbol{u}=v_{\sigma} \boldsymbol{A}^{\sigma}+v_{3} \boldsymbol{A}^{3}-\xi\left(v_{3, \sigma}+v_{\gamma} B_{\sigma}^{\gamma}\right) \boldsymbol{A}^{\sigma}=\left[v_{\sigma}-\xi\left(v_{3, \sigma}+\right.\right.$ $\left.\left.v_{\gamma} B_{\sigma}^{\gamma}\right) \boldsymbol{A}^{\sigma}\right]+v_{3} \boldsymbol{A}^{3}$ and/or $\boldsymbol{u}=v^{\sigma} \boldsymbol{A}_{\sigma}+v^{3} \boldsymbol{A}_{3}-\xi\left(v_{, \sigma}^{3}+v^{\gamma} B_{\gamma}^{\sigma}\right) \boldsymbol{A}_{\sigma}=\left[v^{\sigma}-\xi\left(v_{, \sigma}^{3}+\right.\right.$ $\left.\left.v^{\gamma} B_{\gamma}^{\sigma}\right)\right] \boldsymbol{A}_{\sigma}+v^{3} \boldsymbol{A}_{3}$.

The K-L theory of shells has three unknown variables, namely, $v^{1}, v^{2}, v^{3}$.

\subsection{The displacement field of the Mindlin-Reissner theory of shells}

The Mindlin-Reissner theory of plates is an extension of Kirchhoff-Love plate theory that takes into account shear deformations through-the-thickness of a plate. The theory was proposed in 1951 by R. Mindlin. [14] A similar, but not identical, theory had been proposed earlier by Eric Reissner in 1945.[13] Both theories are intended for thick plates in which the normal to the mid-surface remains straight but not necessarily perpendicular to the mid-surface. The Mindlin-Reissner theory is used to calculate the deformations and stresses in a plate whose thickness is of the order of one tenth the planar dimensions while the Kirchhoff-Love theory is applicable to thinner plates.

Thus $\boldsymbol{u}=\boldsymbol{v}+\xi \boldsymbol{d}=v^{\beta} \boldsymbol{A}_{\beta}+v^{3} \boldsymbol{A}_{3}+\xi d^{\beta} \boldsymbol{A}_{\beta}=\left(v^{\beta}+\xi d^{\beta}\right) \boldsymbol{A}_{\beta}+v^{3} \boldsymbol{A}_{3}$, so we have the components of the arbitrary displacement vector $\boldsymbol{u}=u^{\beta} \boldsymbol{A}_{\beta}+u^{3} \boldsymbol{A}_{3}$ as follows $u^{\beta}=v^{\beta}\left(\xi^{1}, \xi^{2}\right)+\xi d^{\beta}\left(\xi^{1}, \xi^{2}\right)$ and $u^{3}=v^{3}\left(\xi^{1}, \xi^{2}\right)$.

The M-R theory of shells has five unknown variables, namely, $v^{1}, v^{2}, v^{3}, d^{1}, d^{2}$.

\section{Deformation gradient of thin shells}

There are four base vectors in the analysis of shell deformation, namely, $\boldsymbol{A}_{i}, \boldsymbol{a}_{i}, \boldsymbol{G}_{i}, \boldsymbol{g}_{i}$. The relationship of base vectors in both underformed and deformed state can be illustrated in Fig.1. 


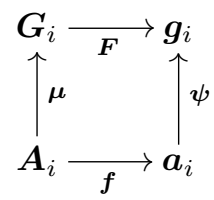

Fig. 1. Relationship of base vectors.

The deformation gradient of the thin shells is defined as

$$
\begin{aligned}
\boldsymbol{F} & =\boldsymbol{g}_{i} \otimes \boldsymbol{G}^{i} \\
& =\boldsymbol{g}_{\alpha} \otimes \boldsymbol{G}^{\alpha}+\boldsymbol{g}_{3} \otimes \boldsymbol{G}^{3} \\
& =\left(\boldsymbol{p}_{, \alpha}+\xi \boldsymbol{\eta}_{, \alpha}\right) \otimes \boldsymbol{G}^{\alpha}+\boldsymbol{\eta} \otimes \boldsymbol{G}^{3} .
\end{aligned}
$$

In the diagram 1, there are four tensors, namely, $\boldsymbol{F}=\boldsymbol{g}_{i} \otimes \boldsymbol{G}^{i}$ is deformation gradient of arbitrary surface, $\boldsymbol{f}=\boldsymbol{a}_{i} \otimes \boldsymbol{A}^{i}$ is middle surface deformation gradient, $\boldsymbol{\mu}=\boldsymbol{G}_{i} \otimes \boldsymbol{A}^{i}$ is shifter tensor from $\boldsymbol{A}_{i}$ to $\boldsymbol{G}_{i}$ in undeformed state, and $\boldsymbol{\psi}=\boldsymbol{g}_{i} \otimes \boldsymbol{a}^{i}$ is shifter tensor from $\boldsymbol{a}_{i}$ to $\boldsymbol{g}_{i}$ in deformed state.

With the help of $\boldsymbol{f}, \boldsymbol{\mu}, \boldsymbol{\psi}$, the deformation gradient $\boldsymbol{F}$ can be expressed in a multiplication format:

$$
\boldsymbol{F}=\boldsymbol{g}_{i} \otimes \boldsymbol{G}^{i}=\left(\boldsymbol{g}_{i} \otimes \boldsymbol{a}^{i}\right) \cdot\left(\boldsymbol{a}_{i} \otimes \boldsymbol{A}^{i}\right) \cdot\left(\boldsymbol{A}_{i} \otimes \boldsymbol{G}^{i}\right)=\boldsymbol{\psi} \cdot \boldsymbol{f} \cdot \boldsymbol{\mu}^{-1} .
$$

The equation (13) indicates that the deformation gradient of shells can be decomposed into the multiplication of middle surface deformation gradient with two shifter tensors $\boldsymbol{\mu}$ and $\boldsymbol{\psi}$. In order words, the study of general shell deformation can be considered as the subject of deformation of the middle surface. This relation can be mathematically interpreted as follows: the middle surface deformation gradient $\boldsymbol{f}$ is push-forwarded to the deformation gradient $\boldsymbol{F}$ by two shifter tensor $\boldsymbol{\mu}$ and $\boldsymbol{\psi}$.

The inverse of shifter tensor $\boldsymbol{\mu}$ is $\boldsymbol{\mu}^{-1}=\boldsymbol{A}_{i} \otimes \boldsymbol{G}^{i}$, namely,

$$
\begin{aligned}
\boldsymbol{\mu}^{-1} & =\boldsymbol{A}_{i} \otimes \boldsymbol{G}^{i} \\
& =\boldsymbol{A}_{\alpha} \otimes \boldsymbol{G}^{\alpha}+\boldsymbol{A}_{3} \otimes \boldsymbol{G}^{3} \\
& =\boldsymbol{A}_{\alpha} \otimes\left(\boldsymbol{A}^{\alpha}-\xi B_{\beta}^{\alpha} \boldsymbol{A}^{\beta}\right)+\boldsymbol{A}_{3} \otimes \boldsymbol{G}^{3} \\
& =\boldsymbol{A}_{\alpha} \otimes \boldsymbol{A}^{\alpha}+\boldsymbol{A}_{3} \otimes \boldsymbol{A}^{3}-\xi \boldsymbol{B} \\
& =\boldsymbol{A}-\xi \boldsymbol{B},
\end{aligned}
$$

where the tensor $\boldsymbol{A}=\boldsymbol{A}_{i} \otimes \boldsymbol{A}^{i}=\boldsymbol{I}$ is an identity tensor, and the 2nd fundamental tensor $\boldsymbol{B}=B_{\beta}^{\alpha} \boldsymbol{A}_{\alpha} \otimes \boldsymbol{A}^{\beta}$.

The deformation gradient of the middle surface $\boldsymbol{f}=\left.\boldsymbol{F}\right|_{\xi=0}$ is given by

$$
\begin{aligned}
\boldsymbol{f} & =\boldsymbol{a}_{i} \otimes \boldsymbol{A}^{i}=\boldsymbol{a}_{\alpha} \otimes \boldsymbol{A}^{\alpha}+\boldsymbol{a}_{3} \otimes \boldsymbol{A}^{3} \\
& =\left(\boldsymbol{A}_{\alpha}+\boldsymbol{v}_{, \alpha}\right) \otimes \boldsymbol{A}^{\alpha}+\boldsymbol{a}_{3} \otimes \boldsymbol{A}^{3} \\
& =\boldsymbol{A}_{\alpha} \otimes \boldsymbol{A}^{\alpha}+\boldsymbol{v}_{, \alpha} \otimes \boldsymbol{A}^{\alpha}+\boldsymbol{a}_{3} \otimes \boldsymbol{A}^{3} .
\end{aligned}
$$




\section{The dislocation density tensor of thin shells}

5.1 The dislocation density tensor on the middle surface

The dislocation density tensor of middle surface is defined as $\overline{\boldsymbol{T}}=\boldsymbol{f} \times \overline{\boldsymbol{\nabla}}$, where the gradient operator $\bar{\nabla}=\nabla_{i} \boldsymbol{A}^{i}=\frac{\partial}{\partial \xi^{\lambda}} \boldsymbol{A}^{\lambda}+\frac{\partial}{\partial \xi} \boldsymbol{A}^{3}$, namely, $\overline{\boldsymbol{T}}=$ $-\frac{1}{2} v^{\alpha} \bar{R}_{\alpha \beta \gamma \omega} \boldsymbol{A}^{\beta} \otimes \boldsymbol{A}^{\gamma} \times \boldsymbol{A}^{\omega}$.

For the two dimensional middle surface, the Riemann tensor has 16 components, in which four are no-zero, namely, $\bar{R}_{1212}=\bar{R}_{2121}=-\bar{R}_{2112}=-\bar{R}_{1221}$. If we denote $\bar{K}$ as a the Gauss curvature of the middle surface, then we have $\bar{R}_{1221}=\bar{K} A$, where $A=\operatorname{det}\left(A_{\alpha \beta}\right)$.

Hence, the dislocation density tensor of the middle surface of the thin shells can be expressed as follows:

$$
\begin{aligned}
\overline{\boldsymbol{T}} & =-\frac{1}{2} v^{\alpha} \bar{R}_{\alpha \beta \gamma \omega} \boldsymbol{A}^{\beta} \otimes \boldsymbol{A}^{\gamma} \times \boldsymbol{A}^{\omega} \\
& =-\frac{1}{2}\left[v^{1} \bar{R}_{1212} \boldsymbol{A}^{2} \otimes \boldsymbol{A}^{1} \times \boldsymbol{A}^{2}+v^{1} \bar{R}_{1221} \boldsymbol{A}^{2} \otimes \boldsymbol{A}^{2} \times \boldsymbol{A}^{1}\right. \\
& \left.+v^{2} \bar{R}_{2112} \boldsymbol{A}^{1} \otimes \boldsymbol{A}^{1} \times \boldsymbol{A}^{2}+v^{2} \bar{R}_{2121} \boldsymbol{A}^{1} \otimes \boldsymbol{A}^{2} \times \boldsymbol{A}^{1}\right] \\
& =-\frac{1}{2} \bar{K} A\left[v^{1} \boldsymbol{A}^{2} \otimes \boldsymbol{A}^{1} \times \boldsymbol{A}^{2}-v^{1} \boldsymbol{A}^{2} \otimes \boldsymbol{A}^{2} \times \boldsymbol{A}^{1}\right. \\
& \left.-v^{2} \boldsymbol{A}^{1} \otimes \boldsymbol{A}^{1} \times \boldsymbol{A}^{2}+v^{2} \boldsymbol{A}^{1} \otimes \boldsymbol{A}^{2} \times \boldsymbol{A}^{1}\right] \\
& =-\bar{K} A\left[v^{1} \boldsymbol{A}^{2} \otimes \boldsymbol{A}^{1} \times \boldsymbol{A}^{2}-v^{2} \boldsymbol{A}^{1} \otimes \boldsymbol{A}^{1} \times \boldsymbol{A}^{2}\right] \\
& =-\bar{K} A\left[v^{1} \boldsymbol{A}^{2} \otimes\left(\boldsymbol{A}^{1} \times \boldsymbol{A}^{2}\right)-v^{2} \boldsymbol{A}^{1} \otimes\left(\boldsymbol{A}^{1} \times \boldsymbol{A}^{2}\right)\right] .
\end{aligned}
$$

Notice $\boldsymbol{A}^{1} \times \boldsymbol{A}^{2}=\sqrt{A} \boldsymbol{A}^{3}$, hence

$$
\begin{aligned}
\overline{\boldsymbol{T}} & =-\bar{K} A\left[v^{1} \boldsymbol{A}^{2} \otimes\left(\sqrt{A} \boldsymbol{A}^{3}\right)-v^{2} \boldsymbol{A}^{1} \otimes\left(\sqrt{A} \boldsymbol{A}^{3}\right)\right] \\
& =-\bar{K} A \sqrt{A}\left[v^{1} \boldsymbol{A}^{2} \otimes \boldsymbol{A}^{3}-v^{2} \boldsymbol{A}^{1} \otimes \boldsymbol{A}^{3}\right] \\
& =\bar{K} A^{3 / 2}\left[v^{2} \boldsymbol{A}^{1} \otimes \boldsymbol{A}^{3}-v^{1} \boldsymbol{A}^{2} \otimes \boldsymbol{A}^{3}\right] .
\end{aligned}
$$

or in component format $\bar{T}_{13}=\bar{K} A^{3 / 2} v^{2}$ and $\bar{T}_{23}=-\bar{K} A^{3 / 2} v^{1}$.

The relation (17) is obtained for the first time by this paper. The (17) clearly shows that the dislocation density tensor $\overline{\boldsymbol{T}}$ of the middle surface is proportional to the Gauss curvature and $A^{3 / 2}$, and has nothing to do with the displacement component $v^{3}$, in other words, the component $v_{3}$ has no any contribution to the $\overline{\boldsymbol{T}}$. 
5.2 The dislocation density tensor on the Mindlin-Reissner theory of shells

Now let us attack the dislocation density tensor $\boldsymbol{T}=-\boldsymbol{F} \times \boldsymbol{\nabla}$, where $\boldsymbol{\nabla}=$ $\nabla_{i} \boldsymbol{G}^{i}=\frac{\partial}{\partial \xi^{\lambda}} \boldsymbol{G}^{\lambda}+\frac{\partial}{\partial \xi} \boldsymbol{G}^{3}$. Hence

$$
\begin{aligned}
\boldsymbol{T}_{(M-R)} & =\left(\boldsymbol{\psi} \boldsymbol{f} \boldsymbol{\mu}^{-1}\right) \times\left(\boldsymbol{G}^{\lambda} \frac{\partial}{\partial \xi^{\lambda}}+\boldsymbol{G}^{3} \frac{\partial}{\partial \xi}\right) \\
& =\frac{\partial\left(\boldsymbol{\psi} \boldsymbol{f} \boldsymbol{\mu}^{-1}\right)}{\partial \xi^{\lambda}} \times \boldsymbol{G}^{\lambda}+\frac{\partial\left(\boldsymbol{\psi} \boldsymbol{f} \boldsymbol{\mu}^{-1}\right)}{\partial \xi} \times \boldsymbol{G}^{3} .
\end{aligned}
$$

If you carry out this calculation, it will generate quite complicated expressions, we are not going to continue this painful calculation. We will find the dislocation density tensor $\boldsymbol{T}$ by the above formula $\boldsymbol{T}=-\frac{1}{2} u^{k} R_{k \beta \gamma \omega} \boldsymbol{G}^{\beta} \otimes \boldsymbol{G}^{\gamma} \times \boldsymbol{G}^{\omega}$, where the Greek indices range over the values 1,2 while the Latin $k$ indices range over the values $1,2,3$.

From previous study of deformation geometry of thin shells, we have the shell displacement vector $\boldsymbol{u}=\boldsymbol{v}+\xi \boldsymbol{d}$.

Hence the dislocation density tensor $\boldsymbol{T}_{(M-R)}$ is given as follows

$$
\begin{aligned}
\boldsymbol{T}_{(M-R)} & =-\frac{1}{2} u^{k} R_{k \beta \gamma \omega} \boldsymbol{G}^{\beta} \otimes \boldsymbol{G}^{\gamma} \times \boldsymbol{G}^{\omega} \\
& =-\frac{1}{2} u^{\alpha} R_{\alpha \beta \gamma \omega} \boldsymbol{G}^{\beta} \otimes \boldsymbol{G}^{\gamma} \times \boldsymbol{G}^{\omega}-\frac{1}{2} u^{3} R_{3 \beta \gamma \omega} \boldsymbol{G}^{\beta} \otimes \boldsymbol{G}^{\gamma} \times \boldsymbol{G}^{\omega} .
\end{aligned}
$$

Notice $R_{3 \beta \gamma \omega}=0$, hence

$$
\begin{aligned}
\boldsymbol{T}_{(M-R)} & =-\frac{1}{2} u^{\alpha} R_{\alpha M C D} \boldsymbol{G}^{M} \otimes \boldsymbol{G}^{C} \times \boldsymbol{G}^{D} \\
& =-\frac{1}{2}\left[u^{1} R_{1212} \boldsymbol{G}^{2} \otimes \boldsymbol{G}^{1} \times \boldsymbol{G}^{2}+u^{1} R_{1221} \boldsymbol{G}^{2} \otimes \boldsymbol{G}^{2} \times \boldsymbol{G}^{1}\right. \\
& \left.+u^{2} R_{2112} \boldsymbol{G}^{1} \otimes \boldsymbol{G}^{1} \times \boldsymbol{G}^{2}+v^{2} R_{2121} \boldsymbol{G}^{1} \otimes \boldsymbol{G}^{2} \times \boldsymbol{G}^{1}\right] .
\end{aligned}
$$

For the two dimensional parallel surface, the Riemann tensor has 16 components, in which four are no-zero, namely, $R_{1212}=R_{2121}=-R_{2112}=-R_{1221}$. If we denote $\bar{K}$ as a the Gauss curvature of the middle surface, then we have $R_{1221}=K G$, where $G=\operatorname{det}\left(G_{\alpha \beta}\right)=A\left(1-2 \xi H+\xi^{2} K\right)^{2}$ and $K=$ $\left(R_{1}+\xi\right)^{-1}\left(R_{2}+\xi\right)^{-1}=\bar{K}\left(1+\xi / R_{1}\right)^{-1}\left(1+\xi / R_{2}\right)^{-1}$, where both $R_{1}, R_{2}$ are the principal radius of curvature of the surface, and the Gauss curvature of the middle surface $\bar{K}=\frac{1}{R_{1} R_{2}}$.

The dislocation density tensor of thin shells can be expressed as follows:

$$
\begin{aligned}
\boldsymbol{T}_{(M-R)} & =K G^{3 / 2}\left[\left(v^{2}+\xi d^{2}\right) \boldsymbol{G}^{1} \otimes \boldsymbol{G}^{3}-\left(v^{1}+\xi d^{1}\right) \boldsymbol{G}^{2} \otimes \boldsymbol{G}^{3}\right] \\
& =\frac{\bar{K} G^{3 / 2}\left[\left(v^{2}+\xi d^{2}\right) \boldsymbol{G}^{1} \otimes \boldsymbol{G}^{3}-\left(v^{1}+\xi d^{1}\right) \boldsymbol{G}^{2} \otimes \boldsymbol{G}^{3}\right]}{\left(1+\xi / R_{1}\right)\left(1+\xi / R_{2}\right)} \\
& \simeq \bar{K} G^{3 / 2} Q\left[\left(v^{2}+\xi d^{2}\right) \boldsymbol{G}^{1} \otimes \boldsymbol{G}^{3}-\left(v^{1}+\xi d^{1}\right) \boldsymbol{G}^{2} \otimes \boldsymbol{G}^{3}\right], \\
& =\bar{K} A^{3 / 2} Q^{4}\left[\left(v^{2}+\xi d^{2}\right) \boldsymbol{G}^{1} \otimes \boldsymbol{G}^{3}-\left(v^{1}+\xi d^{1}\right) \boldsymbol{G}^{2} \otimes \boldsymbol{G}^{3}\right],
\end{aligned}
$$


in which $Q(\xi)=1-2 \xi H+\xi^{2} K, \boldsymbol{G}^{3}=\boldsymbol{A}^{3}=\boldsymbol{A}_{3}$, and $\boldsymbol{G}^{\beta}=\lambda_{\alpha}^{\beta} \boldsymbol{A}^{\alpha}$, where the shifter $\lambda_{\alpha}^{\beta}$ is defined as $\lambda_{\alpha}^{\beta} \mu_{\delta}^{\alpha}=\delta_{\delta}^{\alpha}, \lambda_{\alpha}^{\beta}=\delta_{\alpha}^{\beta}+\xi B_{\alpha}^{\beta}+\xi^{2} B_{\gamma}^{\beta} B_{\alpha}^{\gamma}+\ldots$, hence the metric of the parallel surface is $G^{\alpha \beta}=\boldsymbol{G}^{\alpha} \cdot \boldsymbol{G}^{\beta}=\lambda_{\gamma}^{\alpha} \lambda_{\delta}^{\beta} A^{\gamma \delta}$.

$$
\begin{aligned}
\boldsymbol{T}_{(M-R)} & =\bar{K} A^{3 / 2} Q^{4}\left[\left(v^{2}+\xi d^{2}\right) \boldsymbol{G}^{1} \otimes \boldsymbol{G}^{3}-\left(v^{1}+\xi d^{1}\right) \boldsymbol{G}^{2} \otimes \boldsymbol{G}^{3}\right] . \\
& =\bar{K} A^{3 / 2} Q^{4}\left[\left(v^{2}+\xi d^{2}\right) \lambda_{\alpha}^{1} \boldsymbol{A}^{\alpha} \otimes \boldsymbol{A}^{3}-\left(v^{1}+\xi d^{1}\right) \lambda_{\beta}^{1} \boldsymbol{A}^{\beta} \otimes \boldsymbol{A}^{3}\right] . \\
& (\alpha, \beta=1,2) .
\end{aligned}
$$

The equation (22) is derived for the first time by this paper. The formula shows that the dislocation density tensor of arbitrary surface of thin shells is proportional to $\bar{K} A^{3 / 2}$, and is an order 9 polynomial function of thickness coordinate $\xi$, which can be simplified to a lower order of $\xi$. The equation (22) also indicates that the component $v_{3}$ has no any contribution to the $\boldsymbol{T}_{(M-R)}$.

5.3 The dislocation density tensor of the Kirchhoff-Love theory of thin shells

For Kirchhoff-Love (K-L) theory of thin shells, the rotation vector $\boldsymbol{d}=\boldsymbol{\eta}-$ $\boldsymbol{A}_{3}=\boldsymbol{a}_{3}-\boldsymbol{A}_{3}$, which can be represented by the middle surface displacements $v^{\alpha}$ and thickness-through displacement $v^{3}$, namely, $d^{\sigma}=-\left(v_{, \sigma}^{3}+v^{\gamma} B_{\gamma}^{\sigma}\right)$.

Substitute the $d^{\sigma}$ into the equation (22), therefore the dislocation density tensor $\boldsymbol{T}_{(K-L)}$ of the K-L theory of thin shells can be obtained as follows

$$
\begin{aligned}
\boldsymbol{T}_{(K-L)} & =\bar{K} A^{3 / 2}\left(1-2 \xi H+\xi^{2} K\right)^{4}\left\{\left[v^{2}-\xi\left(v_{, 2}^{3}+v^{\gamma} B_{\gamma}^{2}\right)\right] \lambda_{\alpha}^{1} \boldsymbol{A}^{\alpha} \otimes \boldsymbol{A}^{3}\right. \\
& \left.-\left[v^{1}-\xi\left(v_{, 1}^{3}+v^{\gamma} B_{\gamma}^{1}\right)\right] \lambda_{\beta}^{1} \boldsymbol{A}^{\beta} \otimes \boldsymbol{A}^{3}\right\}, \quad(\alpha, \beta=1,2) .
\end{aligned}
$$

The equation (23) is obtained for the first time by this paper, which shows that the dislocation density tensor of arbitrary surface of thin shells is proportional to $\bar{K} A^{3 / 2}$. The equation (23) reveals that the displacement component $v_{3}$ will have contribution to the $\boldsymbol{T}_{(K-L)}$ because of the Kirchhoff-Love kinematical constraints.

\section{Conclusions}

This paper studied the dislocation density tensor of thin shells. The dislocation density tensor for both Mindlin-Reissner and K-L theory of shells have been explicitly expressed in terms of the Riemann curvature tensor. The formulation reveals that both dislocation density of the shells are proportional to $K A^{3 / 2}$, where $K$ is the Gauss curvature and $A=\operatorname{det}\left(A_{\alpha \beta}\right)$ is the determinant of metric tensor of the middle surface. The study finds that the displacement component $v^{3}$ has no any direct contribution to the dislocation density of the the Mindlin-Reissner theory of shells, but has contribution to the dislocation density of the Kirchhoff-Love theory of shells. The formulations are shown in below table 1 . 
Table 1 Dislocation density tensor of thin shells

\begin{tabular}{ll}
\hline Thin shells & Dislocation density tensor \\
\hline$\overline{\boldsymbol{T}}$ & $\bar{K} A^{3 / 2}\left[v^{2} \boldsymbol{A}^{1} \otimes \boldsymbol{A}^{3}-v^{1} \boldsymbol{A}^{2} \otimes \boldsymbol{A}^{3}\right]$ \\
$\boldsymbol{T}_{(M-R)}$ & $\bar{K} A^{3 / 2}\left(1-2 \xi H+\xi^{2} K\right)^{4}\left[\left(v^{2}+\xi d^{2}\right) \lambda_{\alpha}^{1} \boldsymbol{A}^{\alpha} \otimes \boldsymbol{A}^{3}-\left(v^{1}+\xi d^{1}\right) \lambda_{\beta}^{1} \boldsymbol{A}^{\beta} \otimes \boldsymbol{A}^{3}\right]$ \\
$\boldsymbol{T}_{(K-L)}$ & $\bar{K} A^{3 / 2}\left(1-2 \xi H+\xi^{2} K\right)^{4}\left\{\left[v^{2}-\xi\left(v_{, 2}^{3}+v^{\gamma} B_{\gamma}^{2}\right)\right] \lambda_{\alpha}^{1} \boldsymbol{A}^{\alpha} \otimes \boldsymbol{A}^{3}\right.$ \\
& $\left.-\left[v^{1}-\xi\left(v_{, 1}^{3}+v^{\gamma} B_{\gamma}^{1}\right)\right] \lambda_{\beta}^{1} \boldsymbol{A}^{\beta} \otimes \boldsymbol{A}^{3}\right\}$ \\
\hline
\end{tabular}

\section{Acknowledgements}

This paper is dedicated to the memory of my mentor, Prof. Kaiyuan Ye. Supports by the South Africa National Research Foundation (NRF) (Grant no. 93918) is gratefully acknowledged.

\section{Author contributions statement}

Bohua Sun initiated, completed the project and prepared the manuscript.

\section{The conflict of interest statement}

The author declare no conflict interests with any parties.

\section{References}

1. Aifantis, E.C.: The physics of plastic deformation, Int. J. of Plasticity, Vol. 3, pp. 211-247 (1987).

2. Bilby, B. A. Bullough R. and Smith E.: Continuous distributions of dislocations: a new application of the methods of non-Riemannian geometry, Proceedings of the Royal Society of London A231(1185): 263-273 (1955).

3. Birsan, M. and Neff, P.: On the dislocation density tensor in the Cosserat theory of elastic shells, ArXiv:1602.03421v2 [math-AP] 18 Feb. 2016.

4. Cosserat, E. and Cosserat, F.: Théorie des corps déformables. Hermann et Fils (reprint 2009), Paris (1909).

5. Kondo, K.: Geometry of elastic deformation and incompatibility, Memoirs of the Unifying Study of the Basic Problems in Engineering Science by Means of Geometry, (K. Kondo, ed.), vol. 1, Division C, Gakujutsu Bunken Fukyo-Kai, 1955, pp. 5-17 (1955).

6. Kondo, K.: Non-Riemannien geometry of imperfect crystals from a macroscopic viewpoint, Memoirs of the Unifying Study of the Basic Problems in Engineering Science by Means of Geometry, (K. Kondo, ed.), vol. 1, Division D-I, Gakujutsu Bunken Fukyo-Kai, 1955, pp. 6-17 (1955).

7. Kröner, E: Das Fundamentalintegral der anisotropen elastischen Versetzungsdichte und Spannungsfunktionen, Z. Phys., 142, 463-475 (1955).

8. Kröner, E.: Allgemeine kontinuumstheorie der versetzungen und eigenspannungen. Arch. Rational Mech. Anal., 4(4):273-334 (1960).

9. Le, K.C. and Stumpf, H.: Nonlinear continuum theory of dislocations, Int. J. of Engineering Science 34: 339-358 (1995).

10. Le, K. C. and Stumpf, H.: A model of elastoplastic bodies with continuously distributed dislocations. Int J. of Plasticity 12:611-627 (1996). 
11. Le, K.C. and Gnther, C.: Nonlinear continuum dislocation theory revisited, Int. J. of Plasticity, 53, 164C178 (2014).

12. Le, K.C.: Three-dimensional continuum dislocation theory, Int. J. of Plasticity 76, 213230 (2016).

13. Love, A.E.H.: A Treatise on the Mathematical Theory of Elasticity, Dover, New York (1927).

14. Mindlin, R.D.: Influence of rotatory inertia and shear on flexural motions of isotropic, elastic plates, ASME Journal of Applied Mechanics, Vol. 18 pp. 31C38, 1851.

15. Nye, J. F.: Some geometrical relations in dislocated crystals, Acta Metallurgica 1(2): 153 - 162(1953).

16. Poincaré, H.: Analysis Situs. J. École Polytech. ser 2 1,1-123 (1895).

17. Reissner, E.: The effect of transverse shear deformation on the bending of elastic plates, ASME Journal of Applied Mechanics, Vol. 12, pp. A68-77 (1945).

18. Sun, B.: Compatibility conditions and dislocation density tensor in termas of the Riemann curvature tensor, to Applied Mathemathics and Mechanics (2016).

\section{Appendix 1: Notation, preliminaries on finite deformations}

\subsection{Notations of this paper}

To distinguish the undeformed and deformed configuration, the quantities with the undeformed body (configuration) $\mathfrak{B}$ will be denoted by upper case (majuscules), and those associated with the deformed body (configuration) $\mathfrak{b}$ by lower case (minuscules). When these quantities are referred to Lagrange coordinates $X^{K}$, their indices will be upper case (majuscules); and when they are referred to Euler $x^{k}$, their indices will be by lower case (minuscules). For example, a displacement vector $\boldsymbol{u}$ referred to $X^{K}$ will have components $u_{K}$, and referred to $x^{k}$ will have the components $u_{k}$. If denoting $\boldsymbol{G}_{K}$ and $\boldsymbol{g}_{k}$ as covariant base vector in undeformed body and deformed body, respectively, then we can write the displacement vector in a total form $\boldsymbol{u}=u^{K} \boldsymbol{G}_{K}=u^{k} \boldsymbol{g}_{K}$.

\subsection{Preliminaries on finite deformations}

In undeformed configuration, let $\boldsymbol{X}=\boldsymbol{X}\left(X^{A}\right)$ as position vector of a partcle, $X^{A}$ is Lagrange coordinates of the particle, then its differential is $d \boldsymbol{X}=\frac{\partial \boldsymbol{X}}{\partial X^{A}} d X^{A}=\boldsymbol{X} \boldsymbol{\nabla}_{X^{A}} d X^{A}=$ $\boldsymbol{X} \nabla_{A} d X^{A}=\boldsymbol{G}_{A} d X^{A}$, where $\nabla_{A}=\boldsymbol{G}^{A} \frac{\partial}{\partial X^{A}}$ and $\boldsymbol{G}_{A}$ are covariant derivative and tangent vector in the underformed configuration, respectively. After time $t$, the position moves to deformed configuration, its position is $\boldsymbol{x}=\boldsymbol{x}(\boldsymbol{X}, t)$, its differential is $d \boldsymbol{x}=\frac{\partial \boldsymbol{x}}{\partial x^{i}} d x^{i}=$ $\boldsymbol{x} \boldsymbol{\nabla}_{x^{i}} d x^{i}=\boldsymbol{x} \boldsymbol{\nabla}_{i} d x^{i}=\boldsymbol{g}_{i} d x^{i}$, where $\boldsymbol{\nabla}_{i}=\boldsymbol{g}^{i} \frac{\partial}{\partial x^{i}}$ and $\boldsymbol{g}_{i}$ are gradient operator and tangent vector in the deformed configuration, respectively.

Let $d \boldsymbol{X}$ be line element between two particles $X^{A}$ and $X^{A}+d X^{A}$, after deformation, the line element becomes $d \boldsymbol{x}$ between the corresponding particles $d x^{i}$ and $x^{i}+d x^{i}$, then $d \boldsymbol{x}=\boldsymbol{x}(\boldsymbol{X}+d \boldsymbol{X}, t)-\boldsymbol{x}(\boldsymbol{X}, t)=\frac{\partial \boldsymbol{x}}{\partial \boldsymbol{X}} \cdot d \boldsymbol{X}=\boldsymbol{x} \boldsymbol{\nabla}_{A} \cdot d \boldsymbol{X}=\frac{\partial \boldsymbol{x}}{\partial X^{A}} \frac{\partial X^{A}}{\partial \boldsymbol{X}} \cdot d \boldsymbol{X}=\frac{\partial \boldsymbol{x}}{\partial X^{A}} \boldsymbol{G}^{A} \cdot d \boldsymbol{X}=$ $\frac{\partial \boldsymbol{x}}{\partial x^{i}} \frac{\partial x^{i}}{\partial X^{A}} \boldsymbol{G}^{A} \cdot d \boldsymbol{X}=\boldsymbol{g}_{i} \frac{\partial x^{i}}{\partial X^{A}} \boldsymbol{G}^{A} \cdot d \boldsymbol{X}=\boldsymbol{F} \cdot d \boldsymbol{X}=d \boldsymbol{X} \cdot \boldsymbol{F}^{T}$, where the deformation gradient tensor $\boldsymbol{F}=\frac{\partial \boldsymbol{x}}{\partial \boldsymbol{X}}=\boldsymbol{x} \boldsymbol{\nabla}_{A}=\frac{\partial \boldsymbol{x}}{\partial X^{A}} \boldsymbol{G}^{A}=\boldsymbol{g}_{i} F_{A}^{i} \boldsymbol{G}^{A}=F_{A}^{i} \boldsymbol{g}_{i} \boldsymbol{G}^{A}$, its components $F_{A}^{i}=\frac{\partial x^{i}}{\partial X^{A}}=$ $x_{; A}^{i}=\nabla_{A} x^{i}$, transpose $\boldsymbol{F}^{T}=\frac{\partial \boldsymbol{x}}{\partial X^{A}} \boldsymbol{G}^{A}=F_{A}^{i} \boldsymbol{G}^{A} \boldsymbol{g}_{i}$, and inverse $\boldsymbol{F}^{-1}=\frac{\partial \boldsymbol{X}}{\partial x^{i}} \boldsymbol{g}^{i}=F_{i}^{A} \boldsymbol{G}_{A} \boldsymbol{g}^{i}$.

Let $\boldsymbol{u}$ be displacement vector, then $\boldsymbol{x}=\boldsymbol{u}+\boldsymbol{X}$, then deformation gradient tensor $\boldsymbol{F}=$ $\boldsymbol{I}+\boldsymbol{u} \nabla_{\boldsymbol{X}}=\boldsymbol{I}+\boldsymbol{g}_{i} \boldsymbol{G}^{A} \nabla_{A} u^{i}$, transpose $\boldsymbol{F}^{T}=\boldsymbol{I}+\boldsymbol{\nabla} \boldsymbol{u}=\boldsymbol{I}+\boldsymbol{G}^{A} \boldsymbol{g}_{i} \nabla_{A} u^{i}$ and inverse $\boldsymbol{F}^{-1}=\boldsymbol{I}-\boldsymbol{u} \nabla_{\boldsymbol{x}}=\boldsymbol{I}-\boldsymbol{g}^{j} \boldsymbol{g}^{i} \nabla_{i} u^{j}$

The materials time derivative of $d \boldsymbol{x}$ leads to $d \dot{\boldsymbol{x}}=\dot{\boldsymbol{F}} \cdot d \boldsymbol{X}=\boldsymbol{l F} d \boldsymbol{x}$, in which the velocity gradient tensor is defined as $\boldsymbol{l}=\dot{\boldsymbol{u}} \boldsymbol{\nabla}_{\boldsymbol{x}}=\frac{\partial \dot{\boldsymbol{u}}}{\partial \boldsymbol{x}}=\dot{u}_{; j}^{i} \boldsymbol{g}_{i} \boldsymbol{g}^{j}=\nabla_{j} u^{i} \boldsymbol{g}_{i} \boldsymbol{g}^{j}=\dot{\boldsymbol{F}} \boldsymbol{F}^{-1}$, which can be decomposed as sum of $\boldsymbol{d}$ and $\boldsymbol{w}, \boldsymbol{l}=\boldsymbol{d}+\boldsymbol{w}$, where the rate of deformation tensor $\boldsymbol{d}=\frac{1}{2}\left(\boldsymbol{l}+\boldsymbol{l}^{T}\right)$ and spin tensor $\boldsymbol{w}=\frac{1}{2}\left(\boldsymbol{l}-\boldsymbol{l}^{T}\right)$.

If denote $d \boldsymbol{A}$ as area element in undeformed configuration, then the area in the deformed one $d \boldsymbol{a}=J \boldsymbol{F}^{-T} \cdot d \boldsymbol{A}$ and $d \boldsymbol{A}=J^{-1} \boldsymbol{F}^{T} \cdot d \boldsymbol{a}$, where the Jacobean $J=\operatorname{det}(\boldsymbol{F})$.

The metric tensor in undeformed body $G_{A B}=\boldsymbol{G}_{A} \boldsymbol{G}_{B}$ and in deformed body $g_{i j}=\boldsymbol{g}_{i} \boldsymbol{g}_{j}$. 
The tangent vectors between the undeformed and deformed configuration can be easily transferred as $\boldsymbol{g}_{i}=\delta_{i A} \boldsymbol{G}^{A}, \quad \boldsymbol{g}^{i}=\delta^{i A} \boldsymbol{G}_{A}, \quad \boldsymbol{G}_{A}=\delta_{A i} \boldsymbol{g}^{i}, \quad \boldsymbol{G}^{A}=\delta^{A i} \boldsymbol{g}_{i}$, where the shifters $\delta_{i A}=\boldsymbol{g}_{i} \cdot \boldsymbol{G}_{A}=\delta_{A i}, \quad \delta^{i A^{\prime}}=\boldsymbol{g}^{i} \cdot \boldsymbol{G}^{A}=\delta^{A i}$.

\section{Appendix 2: Introduction of shell geometry}

\subsection{Concepts of shells}

A thin shell/plate is a body that is bounded primarily by two closely spaced curved surfaces with boundary. The geometric feature of the shells is that the thickness length scale is much smaller compare with other two dimensions. This feature make the shells can easily be bended, which make one surface in stretching another in compression. This antisymmetrical deformation respect to the middle surface will have a possibility to generate cracks on the tension surface.

The distance in between the curved surfaces is called the thickness of the shell $h\left(\xi^{1}, \xi^{2}\right)$. The surface in the middle of the curved surface is called the middle surface of the shell. The middle surface in undeformed configuration can be expressed by the parametric representation $\boldsymbol{P}=\boldsymbol{P}\left(\xi^{1}, \xi^{2}\right), \quad\left(\xi^{1}, \xi^{2}\right) \in \Omega$, where $\boldsymbol{P}$ is the position vector from the origin $O$ to points on the mid-surface, and the domain of the definition of the parameters is a closed region $\Omega$ in the $\left(\xi^{1}, \xi^{2}\right)$-plane. We assume that there is one-to-one mapping between the pairs of numbers $\left(\xi^{1}, \xi^{2}\right) \in \Omega$ and the points of the mid-surface.

Let $\boldsymbol{A}_{3}=\boldsymbol{A}_{3}\left(\xi^{1}, \xi^{2}\right)$ the unit normal vector on the middle surface, then the points out of the mid-surface in the shell are given by the position vectors $\boldsymbol{X}\left(\xi^{1}, \xi^{2}, \xi^{3}\right)=\boldsymbol{P}\left(\xi^{1}, \xi^{2}\right)+$ $\xi \boldsymbol{A}_{3}\left(\xi^{1}, \xi^{2}\right)$, where $\xi^{3}$ is thick-through-coordinate, so that $\xi^{3}=0$ is the middle surface.

We assume that $\frac{1}{2} h<\left|R_{\min }\right| \neq 0$, where $R_{\min }$ is the numerically smallest radius of curvature of the middle surface. A shell is called thin when the thickness is small compact with the other dimensions of the shell.

2.2 Differential geometry of shell surfaces in the undeformed state

It is known that the parameters $\left(\xi^{1}, \xi^{2}\right)$ are called curvilinear coordinates of the surfaces, and the $\xi^{1}$ and $\xi^{2}$ are called the coordinate curves.

The covariant base vectors $\boldsymbol{A}_{\alpha}$ of the surface are defined by $\boldsymbol{A}_{\alpha}=\boldsymbol{P}_{, \alpha}=\frac{\partial \boldsymbol{P}}{\partial \xi^{\alpha}}$, where the Greek subscripts represent the numbers 1,2. The infinitesimal vector connecting two points on the surface with coordinates $\xi^{\alpha}$ and $\xi^{\alpha}+d \xi^{\alpha}$ is given by $d \boldsymbol{P}=\boldsymbol{P}_{, \alpha} d \xi^{\alpha}=\boldsymbol{A}_{\alpha} d \xi^{\alpha}$. The length $d L$ of this vector is therefore determined by $(d L)^{2}=d \boldsymbol{P} \cdot d \boldsymbol{P}=\boldsymbol{A}_{\alpha} \cdot \boldsymbol{A}_{\beta} d \xi^{\alpha} d \xi^{\beta}=$ $A_{\alpha \beta} d \xi^{\alpha} d \xi^{\beta}$, which is the first fundamental form of the surface and $A_{\alpha \beta}=\boldsymbol{A}_{\alpha} \cdot \boldsymbol{A}_{\beta}$ is metric tensor of the surface. The surface area $d \Sigma$ is given by $d \Sigma=\sqrt{A} d \xi^{1} d \xi^{2}, \quad A=\operatorname{det}\left(A_{\alpha \beta}\right)$. The contra-variant base vectors of the middle surface are given by $\boldsymbol{A}^{\alpha}=A^{\alpha \beta} \boldsymbol{A}_{\beta}$, where $A^{\alpha \beta}=\boldsymbol{A}^{\alpha} \cdot \boldsymbol{A}^{\beta}$ and $\boldsymbol{A}^{\alpha} \cdot \boldsymbol{A}_{\beta}=\delta_{\beta}^{\alpha}$.

The unit normal vector $\boldsymbol{A}_{3}$ is defined as $\boldsymbol{A}_{3}=\frac{1}{\sqrt{A}} \boldsymbol{A}_{1} \times \boldsymbol{A}_{2}$, and $\boldsymbol{A}_{3} \cdot \boldsymbol{A}_{\alpha}=0$ and $\boldsymbol{A}_{3} \cdot \boldsymbol{A}^{\alpha}=0$. If we introduce two dimensional permutation tensor $e_{\alpha \beta}$ and $e^{\alpha \beta}$, then we have following useful relations $\boldsymbol{A}_{\alpha} \times \boldsymbol{A}_{\beta}=e_{\alpha \beta} \boldsymbol{A}_{3}, \quad \boldsymbol{A}^{\alpha} \times \boldsymbol{A}^{\beta}=e^{\alpha \beta} \boldsymbol{A}_{3}, \boldsymbol{A}_{3} \times \boldsymbol{A}_{\alpha}=$ $e_{\alpha \beta} \boldsymbol{A}^{\beta}, \quad \boldsymbol{A}_{3} \times \boldsymbol{A}^{\alpha}=e^{\alpha \beta} \boldsymbol{A}_{\beta}$, where the permutation tensors are given by $e_{\alpha \beta}: e_{12}=$ $-e_{21}=\sqrt{A}, \quad e_{11}=e_{22}=0 ; \quad e^{\alpha \beta}: \quad e_{12}=-e_{21}=1 / \sqrt{A}, \quad e^{11}=e^{22}=0$, and $e-\delta$ identity $e^{\alpha \lambda} e_{\beta \mu}=\delta_{\beta}^{\alpha} \delta_{\mu}^{\lambda}-\delta_{\mu}^{\alpha} \delta_{\beta}^{\lambda}$.

Differentiating $\boldsymbol{A}_{3} \cdot \boldsymbol{A}_{\beta}=0$ with respect to $\xi_{\alpha}$ we get $\boldsymbol{A}_{3, \alpha} \cdot \boldsymbol{A}_{\beta}+\boldsymbol{A}_{3} \cdot \boldsymbol{A}_{\beta, \alpha}=0$. The second fundamental tensor $B_{\alpha \beta}$ is defined by $B_{\alpha \beta}=-A_{3, \alpha} \cdot \boldsymbol{A}_{\beta}=\boldsymbol{A}_{3} \cdot \boldsymbol{A}_{\beta, \alpha}=\boldsymbol{A}_{3} \cdot \boldsymbol{A}_{\alpha, \beta}=B_{\beta \alpha}$. If we introduce the Christofell connection $\Gamma_{\alpha \beta}^{\lambda}$, the we have derivatives of the base vectors as follows $\boldsymbol{A}_{\alpha, \beta}=\boldsymbol{A}_{\beta, \alpha}=\Gamma_{\alpha \beta}^{\lambda} \boldsymbol{A}_{\lambda}+b_{\alpha \beta} \boldsymbol{A}_{3}, \quad \boldsymbol{A}_{3, \alpha}=-B_{\alpha}^{\beta} \boldsymbol{A}_{\beta}$, and derivatives of the base vectors $A_{, \beta}^{\alpha}=-\Gamma_{\lambda \beta}^{\alpha} A^{\lambda}+b_{\beta}^{\alpha} A_{3}$. If introduce the covariant derivatives of the base vectors $\boldsymbol{A}_{\alpha \mid \beta}=\boldsymbol{A}_{\alpha, \beta}-\Gamma_{\alpha \beta}^{\lambda} \boldsymbol{A}_{\lambda}$, then we have $\boldsymbol{A}_{\alpha \mid \beta}=B_{\alpha \beta} \boldsymbol{A}_{3}, \quad A_{3 \mid \alpha}=-B_{\alpha}^{\beta} \boldsymbol{A}_{\beta}$.

If we assume that the coordinate curves are the lines of curvature. This system of coordinate curves has particularly simple properties, so that the equations of the theory of shells, when written in full, assume a relatively simple form in this system. On the other hand, the sue of the special curvilinear coordinates implies a certain restriction on the field 
of application of the theory. In order to be able required and the determination of these curves for a given surface is, in general, a fairly complicated problem. However, for many of the types of shells used in practice, the geometry of the middle surface is of a simple nature (e.g. surfaces of revolution or cylindrical surfaces), so that the lines of curvature are already known. In such cases the following formulae can be used directly.

As the coordinate curves are lines of curvature, the $\xi^{1}$ and $\xi^{2}$ curves are mutually orthogonal families of curve. It follows that $\boldsymbol{A}_{1} \cdot \boldsymbol{A}_{2}=0$. The length of the base vectors ar denoted by $A_{(1)}$ and $A_{(2)}$, and we have $A_{(\alpha)}=\left|\boldsymbol{A}_{(\alpha)}\right|=\sqrt{\boldsymbol{A}_{\alpha} \cdot \boldsymbol{A}_{\alpha}}$, which is called Lamé parameters of the surface. By thie definition, we fnd for the length of $d L_{(\alpha)}$ of line elements along the coordinate curves $\left(d L_{(\alpha)}\right)^{2}=\boldsymbol{A}_{\alpha} \cdot \boldsymbol{A}_{\alpha}\left(d \xi_{\alpha}\right)^{2}=A_{\alpha}^{2} d \xi_{\alpha}^{2}$, namely, $d L_{(\alpha)}=A_{\alpha} d \xi_{\alpha}$. The area $d \Sigma$ of a small surface bounded by coordinate curves with side lengths $d L_{(1)}$ and $d L_{(2)}$ is given by $d \Sigma=d L_{(1)} d L_{(2)}=A_{1} A_{2} d \xi_{1} d \xi_{2}$.

Arbitrary parallel surface is the surface with distance $\xi$ to the middle surface. The position in this surface will be $\boldsymbol{X}=\boldsymbol{P}+\xi \boldsymbol{A}_{3}$, accordingly, the corresponding quantities can be defined and listed in the table 2 .

Table 2 Geometry of shell surface in undeformed state

\begin{tabular}{c|c|c}
\hline & middle surface & parallel surface of middle surface \\
& $\xi=0$ & $\xi \neq 0$ \\
\hline Position vector & $\boldsymbol{P}$ & $\boldsymbol{X}$ \\
Line element & $d \boldsymbol{P}$ & $d \boldsymbol{X}$ \\
Base vector & $\boldsymbol{A}_{\alpha}, \boldsymbol{A}^{\alpha}, \boldsymbol{A}_{3}=\boldsymbol{A}^{3}$ & $\boldsymbol{G}_{\alpha}, \boldsymbol{G}^{\alpha}, \boldsymbol{G}_{3}=\boldsymbol{G}^{3}$ \\
& $\boldsymbol{A}_{\alpha}=\frac{\partial \boldsymbol{P}}{\partial \xi^{\alpha}}=\boldsymbol{P}_{, \alpha}$ & $\boldsymbol{G}_{\alpha}=\frac{\partial \boldsymbol{X}}{\partial \xi^{\alpha}}=\boldsymbol{X}_{, \alpha}$ \\
Unit normal vector & $\boldsymbol{A}_{3}=\frac{1}{\sqrt{A}} \boldsymbol{A}_{1} \times \boldsymbol{A}_{2}$ & $\boldsymbol{G}_{3}=\frac{1}{\sqrt{G}} \boldsymbol{G}_{1} \times \boldsymbol{G}_{2}$ \\
Lamé parameters & $A_{(\alpha)}=\left(\boldsymbol{A}_{\alpha} \cdot \boldsymbol{A}_{\alpha}\right)^{1 / 2}$ & $G_{(\alpha)}=\left(\boldsymbol{G}_{\alpha} \cdot \boldsymbol{G}_{\alpha}\right)^{1 / 2}$ \\
1st fund. form & $d \boldsymbol{P} \cdot d \boldsymbol{P}=A_{\alpha \beta} d \xi^{\alpha} d \xi^{\beta}$ & $d \boldsymbol{X} \cdot d \boldsymbol{X}=G_{\alpha \beta} d \xi^{\alpha} d \xi^{\beta}$ \\
& $A_{\alpha \beta}=\boldsymbol{A}_{\alpha} \cdot \boldsymbol{A}_{\beta}$ & $G_{\alpha \beta}=\boldsymbol{G}_{\alpha} \cdot \boldsymbol{G}_{\beta}$ \\
2nd fund. form & $d \boldsymbol{P} \cdot d \boldsymbol{A}_{3}=B_{\alpha \beta} d \xi^{\alpha} d \xi^{\beta}$ & $d \boldsymbol{X} \cdot d \boldsymbol{G}_{3}=C_{\alpha \beta} d \xi^{\alpha} d \xi^{\beta}$ \\
& $B_{\alpha \beta}=-\boldsymbol{A}_{3, \alpha} \cdot \boldsymbol{A}_{\beta}=\boldsymbol{A}_{3} \cdot \boldsymbol{A}_{\alpha, \beta}$ & $C_{\alpha \beta}=-\boldsymbol{G}_{3, \alpha} \cdot \boldsymbol{G}_{\beta}=\boldsymbol{G}_{3} \cdot \boldsymbol{G}_{\alpha, \beta}$ \\
\hline
\end{tabular}

The base vectors in the undeformed state $\boldsymbol{G}_{3}=\boldsymbol{X}_{, \xi}=\boldsymbol{A}_{3}$, and $\boldsymbol{G}_{\alpha}=\boldsymbol{X}_{, \alpha}=\boldsymbol{P}_{, \alpha}+$ $\xi \boldsymbol{A}_{3, \alpha}=\boldsymbol{A}_{\alpha}+\xi \boldsymbol{A}_{3, \alpha}=\boldsymbol{A}_{\alpha}-\xi B_{\alpha}^{\beta} \boldsymbol{A}_{\beta}=\mu_{\alpha}^{\beta} \boldsymbol{A}_{\beta}=\mu_{\alpha \beta} \boldsymbol{A}^{\beta}$, where the shiter tensor $\mu_{\alpha \beta}=$ $A_{\alpha \beta}-\xi B_{\alpha \beta}, \mu_{\alpha}^{\beta}=\delta_{\alpha}^{\beta}-\xi B_{\alpha}^{\beta}$, and metric tensor $G_{\alpha \beta}=\mu_{\alpha}^{\lambda} \mu_{\lambda \beta}=A_{\lambda \rho} \mu_{\alpha}^{\lambda} \mu_{\beta}^{\rho}, G_{\alpha 3}=0, G_{33}=$ 1. $\sqrt{G / A}=\frac{1}{2} \delta_{\rho \mu}^{\alpha \beta} \mu_{\alpha}^{\rho} \mu_{\beta}^{\mu}=1-2 \xi H+(\xi)^{2} K$, mean curvature $H=\frac{1}{2} B_{\alpha}^{\alpha}=\frac{1}{2}\left(B_{1}^{1}+B_{2}^{2}\right)$, the Gauss curvature $K=\frac{1}{2} \delta_{\rho \mu}^{\alpha \beta} B_{\alpha}^{\rho} B_{\beta}^{\mu}=B_{1}^{1} B_{2}^{2}-B_{2}^{1} B_{1}^{2}$.

2.3 Differential geometry of shell surfaces in the deformed state

All quantities in the deformed state are listed in the table 3. Consider an arbitrary displacement $\boldsymbol{v}\left(\xi^{1}, \xi^{2}\right)$ of the middle surface, then we have new position vector of middle surface $\boldsymbol{p}=\boldsymbol{P}+\boldsymbol{v}$, base vector of deformed middle surface $\boldsymbol{a}_{\alpha}=\boldsymbol{A}_{\alpha}+\boldsymbol{v}, \alpha, \quad \boldsymbol{a}_{3}=\boldsymbol{a}^{3}$. 
Table 3 Geometry of shell surface in deformed state

\begin{tabular}{c|c|c}
\hline & middle surface & parallel surface of middle surface \\
& $\xi=0$ & $\xi \neq 0$ \\
\hline Position vector & $\boldsymbol{p}$ & $\boldsymbol{x}$ \\
Line element & $d \boldsymbol{p}$ & $d \boldsymbol{x}$ \\
Base vector & $\boldsymbol{a}_{\alpha}, \boldsymbol{a}^{\alpha}, \boldsymbol{a}_{3}=\boldsymbol{a}^{3}$ & $\boldsymbol{g}_{\alpha}, \boldsymbol{g}^{\alpha}, \boldsymbol{g}_{3}=\boldsymbol{g}^{3}$ \\
& $\boldsymbol{a}_{\alpha}=\frac{\partial \boldsymbol{p}}{\partial \xi^{\alpha}}=\boldsymbol{p}_{, \alpha}$ & $\boldsymbol{g}_{\alpha}=\frac{\partial \boldsymbol{x}}{\partial \xi^{\alpha}}=\boldsymbol{x}, \alpha$ \\
Unit normal vector & $\boldsymbol{a}_{3}=\frac{1}{\sqrt{a}} \boldsymbol{a}_{1} \times \boldsymbol{a}_{2}$ & $\boldsymbol{g}_{3}=\frac{1}{\sqrt{g}} \boldsymbol{g}_{1} \times \boldsymbol{g}_{2}$ \\
Lamé parameters & $a_{(\alpha)}=\left(\boldsymbol{a}_{\alpha} \cdot \boldsymbol{a}_{\alpha}\right)^{1 / 2}$ & $g_{(\alpha)}=\left(\boldsymbol{g}_{\alpha} \cdot \boldsymbol{g}_{\alpha}\right)^{1 / 2}$ \\
1st fund. form & $d \boldsymbol{p} \cdot d \boldsymbol{p}=a_{\alpha \beta} d \xi^{\alpha} d \xi^{\beta}$ & $d \boldsymbol{x} \cdot d \boldsymbol{x}=g_{\alpha \beta} d \xi^{\alpha} d \xi^{\beta}$ \\
& $a_{\alpha \beta}=\boldsymbol{a}_{\alpha} \cdot \boldsymbol{a}_{\beta}$ & $g_{\alpha \beta}=\boldsymbol{g}_{\alpha} \cdot \boldsymbol{g}_{\beta}$ \\
2nd fund. form & $d \boldsymbol{p} \cdot d \boldsymbol{a}_{3}=b_{\alpha \beta} d \xi^{\alpha} d \xi^{\beta}$ & $d \boldsymbol{x} \cdot d \boldsymbol{g}_{3}=c_{\alpha \beta} d \xi^{\alpha} d \xi^{\beta}$ \\
& $b_{\alpha \beta}=-\boldsymbol{a}_{3, \alpha} \cdot \boldsymbol{a}_{\beta}=\boldsymbol{a}_{3} \cdot \boldsymbol{a}_{\alpha, \beta}$ & $c_{\alpha \beta}=-\boldsymbol{g}_{3, \alpha} \cdot \boldsymbol{g}_{\beta}=\boldsymbol{g}_{3} \cdot \boldsymbol{g}_{\alpha, \beta}$ \\
\hline
\end{tabular}

